\title{
M860, a Monoclonal Antibody against Human Lactoferrin, Enhances Tumoricidal Activity of Low Dosage Lactoferrin via Granzyme B Induction
}

\author{
Ya Li ${ }^{1,2}{ }^{,}$Jie Li ${ }^{1}$, Zheng Gong ${ }^{1}$, Xiao-Hua Pan ${ }^{1}$, Zi-Han Ma ${ }^{1}$, Shu-Yan Ma ${ }^{1}$, Hong-Min Wang ${ }^{1}$, \\ Hong-Liang Dong ${ }^{1}$, Fang-Yuan Gong ${ }^{1,2, *}$ and Xiao-Ming Gao ${ }^{1,2, *}$ \\ 1 The Institute of Biology and Medical Sciences, Soochow University, Suzhou 215123, China \\ 2 Key Laboratory of Systemic Biomedicine of Suzhou, Suzhou 215000, China \\ * Correspondence: gongfangyuan@suda.edu.cn (F.-Y.G.); xmgao@suda.edu.cn (X.-M.G.); \\ Tel./Fax: +86-512-65881256 (F.-Y.G. \& X.-M.G.)
}

Received: 9 August 2019; Accepted: 2 October 2019; Published: 9 October 2019

\begin{abstract}
Lactoferrin (LF) is a soluble glycoprotein of the transferring family found in most biological fluids, functioning as a major first line defense molecule against infection in mammals. It also shows certain anti-tumor activity, but its clinical application in tumor therapy is limited because high dosage is required. In this study, we demonstrate that M860, a monoclonal antibody against human LF (hLF), could significantly increase the anti-tumor potential of low dosage hLF by forming LF-containing immune complex (IC). Human monocytes primed with LF-IC, but not hLF or M860 alone, or control ICs, showed strong tumoricidal activity on leukemia cell lines Jurkat and Raji through induction of secreted Granzyme B (GzB). LF-IC is able to colligate membrane-bound CD14 (a TLR4 co-receptor) and Fc $\gamma$ RIIa (a low affinity activating Fc $\gamma$ receptor) on the surface of human monocytes, thereby triggering the Syk-PI3K-AKT-mTOR pathway leading to GzB production. Our work identifies a novel pathway for LF-mediated tumoricidal activity and may extend the clinical application of LF in tumor therapy.
\end{abstract}

Keywords: Lactoferrin Immune-Complex; Granzyme B; Monocytes; tumoricidal activity

\section{Introduction}

Lactoferrin (LF), an 80-kDa glycoprotein with iron-binding ability, was discovered in mammary secretions and also in the second granules of neutrophils, and could be extracellularly released under conditions of stress or inflammation [1]. It functions as a major defense molecule against infections, and also exhibits certain anti-tumor potential both in vitro and in vivo [2,3]. It has been reported that down-regulation of LF gene in cells leads to an increase of malignant tumors [4,5]. LF and its derivatives induced apoptosis of tumor cells through both extrinsic and intrinsic pathways [3,6]. LF could also inhibit phosphorylation of AKT thus inducing cell cycle arrest of tumor cells [7,8]. In addition to the direct function of LF on tumor cells, LF could also affect immune cells to evade cancers. Bovine LF could significantly improve the anti-tumor activity of NK cells and macrophages [9,10]. As a multifunctional protein, LF is widely used for various clinical conditions. For instance, it is utilized for treating stomach and intestinal ulcers [11,12]. It is also applied as an antioxidant to protect against infections or tissue damage associated with aging [13-15]. However, clinical application of LF in tumor therapy is limited because of fast clearance of LF in vivo and high dosage requirement for effective therapy $[16,17]$.

M860, an anti-human LF (hLF) monoclonal antibody produced in this laboratory, can form immune-complex (IC) with hLF [18]. We have previously demonstrated that LF-IC (M860+hLF) was very potent in inducing pro-inflammatory cytokines including TNF $\alpha$ by human monocytes/macrophages [19]. 
Tumor-associated macrophages (TAM) are mostly of M2 phenotype and capable of promoting tumor growth by secreting immune-suppressive factors [20]. Interestingly, LF-IC could drive M2 to M1 conversion, implying a possible anti-tumor effect of this unique IC [21].

Monocytes have recently emerged as important regulators of cancer development and progression [22,23], through phagocytosis or direct killing of tumor cells, secretion of tumoricidal mediators and differentiation into tyrosine-based activation motifs (TAMs) [24,25]. When exposed to IFN- $\gamma$ or IFN- $\alpha$, monocytes could produce soluble protein TRAIL, thereby inducing cell death in cancer cells [26]. On the other hand, however, monocytes could also secrete factors that promote tumor cell invasion [27]. Therefore, monocytes exhibit Janus effects on tumorigenesis. Setting monocytes in an anti-tumor mode represents a useful strategy for treatment against malignancies in vivo. In the present study, we investigate whether and how low combination of M860 and dose hLF could render human monocytes strong tumoricidal activity.

\section{Results}

\subsection{Human Monocytes Acquire Potent Tumoricidal Activity Following LF-IC Stimulation}

Based upon our previous findings that LF-IC induces a large amount of TNF- $\alpha$ production by human monocytes via co-ligation of CD14/TLR4 and Fc $\gamma$ RIIa (CD32a) [19], we hypothesized that LF-IC might be able to render human monocytes tumoricidal activity due to its Cd14/TLR4-Fc $\gamma$ RIIa co-ligation property. Freshly purified human blood monocytes were primed for $24 \mathrm{~h}$ with either LF-IC (M860+LF), or hLF, or M860, or complex between chicken ovalbumin (OVA) and OVA-specific mouse mAb M562 (OVA-IC), followed by evaluation for ability to kill leukemia line cells of Jurkat and Raji in vitro. Figs. 1A \& 1B show that human monocytes acquired potent tumoricidal activity after treatment with LF-IC, but not with hLF or M860 alone, or OVA-IC, or OVA-IC+hLF. The mAb M562 in OVA-IC is also of mouse IgG1 isotype [19], which is expected to bind Fc $\gamma$ Rs on monocyte surface in the same manner as M860, yet OVA-IC was unable to activate human monocytes. This is likely due to the lack of CD14/TLR-binding ability of OVA-IC. Importantly, a mixture of hLF and OVA-IC, which was expected to activate both CD14/TLR4 as well as Fc $\gamma$ Rs, did not show positive effects, indicating that the CD14/TLR4-Fc $\gamma$ RIIa co-ligation property of LF-IC is of critical importance for its anti-tumor potential.

Next we addressed the question if the tumoricidal effect of LF-IC-stimulated monocytes was mediated by soluble factors using transwell assays. Primary human monocytes and CFSE-labeled Jurkat, or Raji, cells were cultured in lower and upper (insert) wells, respectively, of transwell chambers for $18 \mathrm{~h}$ in the presence of LF-IC. This system prevents direct cell-cell contact but allows tumor cells access to soluble factors secreted by responder monocytes in the opposite wells of the same chambers. Results presented in Figure 1C,D indicate that supernatant from the LF-IC-stimulated human monocytes was sufficient to cause tumor cell death, indicating that the tumoricidal function of LF-IC-primed monocytes was mediated via soluble factors and independent of direct cell-cell contact.

\subsection{The Tumoricidal Function of LF-IC-Primed Human Monocytes Is Independent of TNF $\alpha$ Production}

$\mathrm{TNF} \alpha$ is known to trigger apoptosis in tumor cells through blocking NF- $\mathrm{kB}$ signaling [28,29], and thus considered a possible mediator of the tumoricidal effect of LF-IC-primed monocytes. However, Etanercept, a blocking $\mathrm{mAb}$ against $\mathrm{TNF} \alpha$, did not affect the tumoricidal activity of LF-IC primed monocytes (Figure 2), arguing for the presence of soluble factors other than TNF $\alpha$ responsible for the killing of the tumor cells in these experiments. 
A

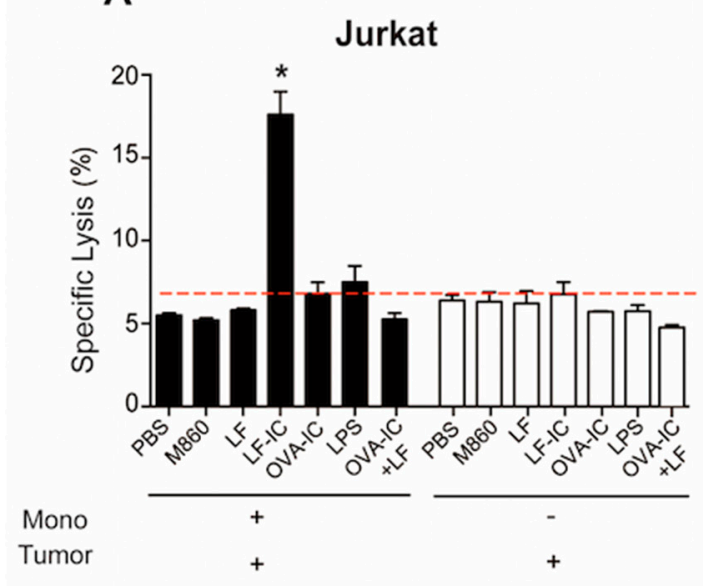

C

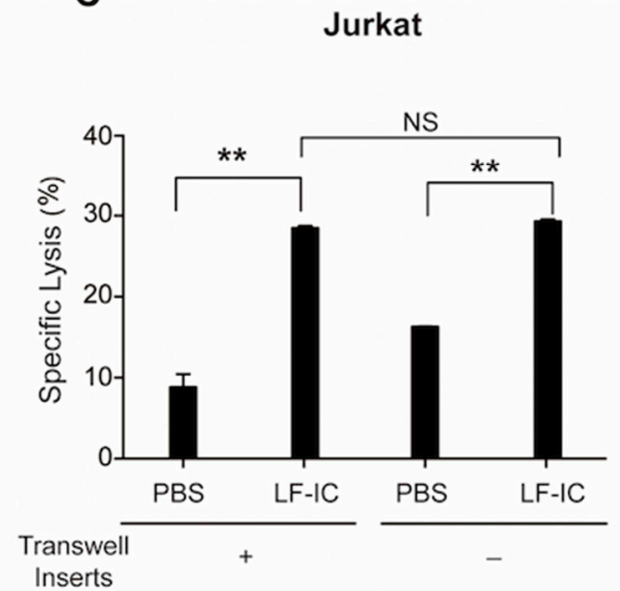

B

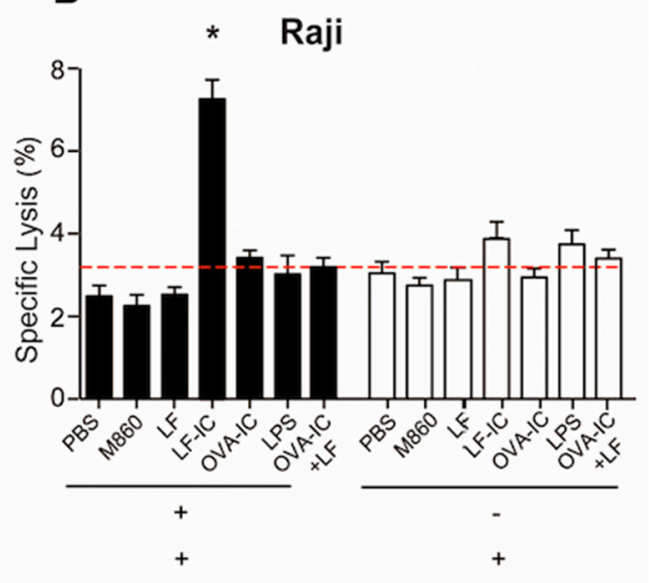

D

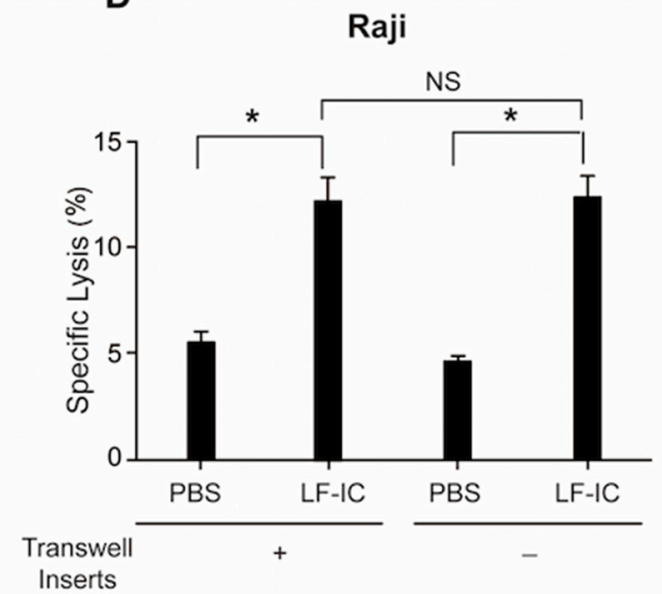

Figure 1. Human monocytes acquire tumoricidal activity after LF-IC stimulation. (A,B) Freshly purified human monocytes were primed with 30 g/mL LF, M860, LF-IC (M860+LF), OVA-IC (OVA+M562), OVA-IC +LF or $10 \mathrm{ng} / \mathrm{mL}$ LPS for $24 \mathrm{~h}$. CFSE labeled Jurkat (A) or Raji (B) were then added and incubated for $18 \mathrm{~h}$, with Jurkat or Raji cells treated with LF, M860, LF-IC, OVA-IC as negative controls. (C,D) Freshly purified human monocytes were primed with $30 \mu \mathrm{g} / \mathrm{mL}$ LF-IC. CFSE labeled Jurkat or Raji cells were seeded directly to the culture or were placed in transwell inserts. Cells were acquired and analyzed by Flow Cytometry with Propidium Iodide staining post co-culture for $18 \mathrm{~h}$. Specific Lysis were expressed by the percentage of $\mathrm{CFSE}^{+} \mathrm{PI}^{+}$cell counts in $\mathrm{CFSE}^{+}$cell counts. ${ }^{*} p<0.05,{ }^{* *} p<0.01$.

\subsection{Granzyme B Is a Key Mediator of the Tumoricidal Function of LF-IC-Primed Monocytes}

Based on our analysis on the differentially expressed genes (DEGs) of RNA-seq data from LF-ICand OVA-IC-primed human monocytes [30], Granzyme B (GzB), a potent cytotoxic protein produced by myeloid cells [31,32], was amongst the most significantly up-regulated genes in LF-IC-primed cells, which was confirmed by q-PCR (Figure 3A) and ELISA (Figure 3B) results. Furthermore, concentration of GzB in supernatant of LF-IC-stimulated monocyte cultures reached plateau by $48 \mathrm{~h}$ (Figure 3C). It has also been reported that Z-AAD-CMK could bind to GzB and irreversibly inhibit its cytotoxic activity. Consistently, the tumoricidal activity of LF-IC-primed human monocytes was dose-dependently inhibited by Z-AAD-CMK (Figs. 3D\& 3E). Since tumor cells are known to polarize monocytes through secreted soluble factors or exosomes [20], Jurkat or Raji cells might also be able to induce GzB expression by human monocytes. However, concentration of GzB in the supernatant of LF-IC primed monocyte cultures was unaffected by the presence of these tumor cells (Figure 3F). These results together confirm an indispensable role for GzB in the tumoricidal function of LF-IC-primed monocytes. 
A

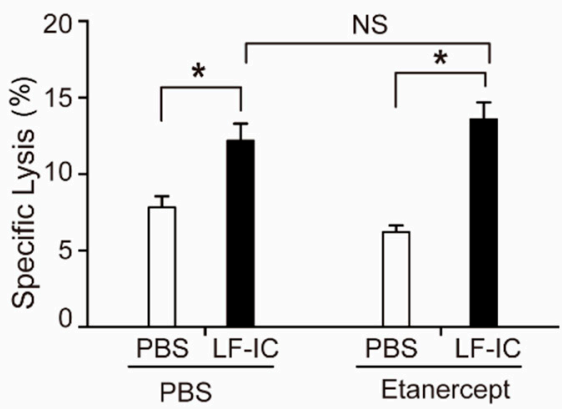

B

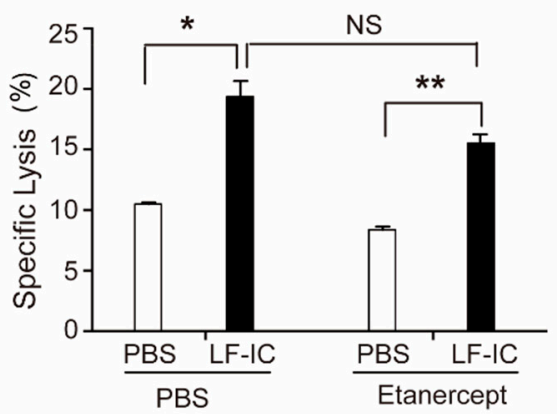

Figure 2. The tumoricidal function of LF-IC-primed human monocytes is independent of TNF $\alpha$ secretion. LF-IC primed monocytes were treated by $100 \mu \mathrm{g} / \mathrm{mL}$ Etanercept or left untreated for $24 \mathrm{~h}$. CFSE labeled Jurkat (A) or Raji (B) were then added and co-cultured with primed monocytes for $18 \mathrm{~h}$. Cells were acquired and analyzed by FACS with PI staining. Results were expressed by the percentage of $\mathrm{CFSE}^{+} \mathrm{PI}^{+}$cell counts in $\mathrm{CFSE}^{+}$cell counts. ${ }^{*} p<0.05,{ }^{* *} p<0.01$.

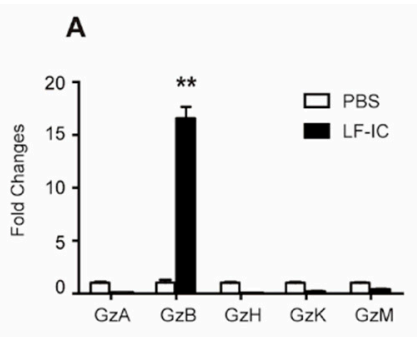

D

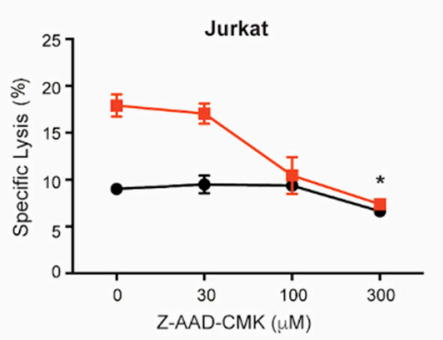

B

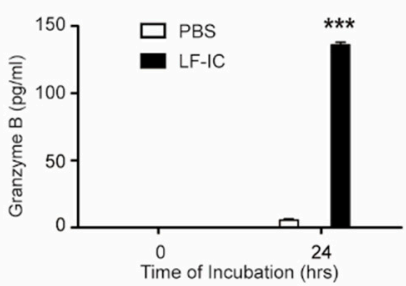

E

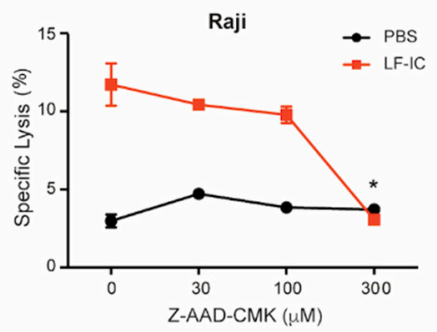

C

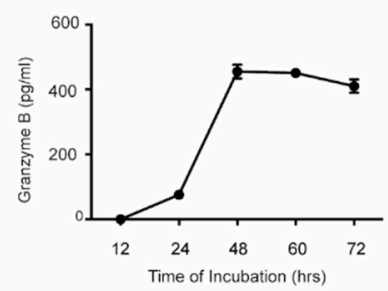

F

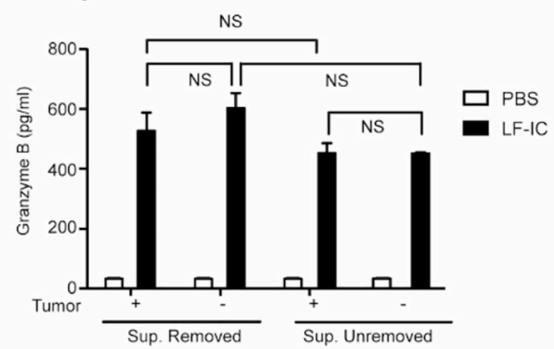

Figure 3. The tumoricidal function of LF-IC-primed human monocytes is dependent on Granzyme B production. Freshly purified human monocytes were primed with $30 \mu \mathrm{g} / \mathrm{mL} \mathrm{M860-IC} \mathrm{for} 24 \mathrm{~h}$. RNA were extracted and expression of Granzyme family were tested by q-PCR (A). GzB in Supernatants was determined by ELISA (B). (C) Freshly purified human monocytes were primed with $30 \mu \mathrm{g} / \mathrm{mL}$ LF-IC for 12, 24, 48, 60, 72 h. GzB in Supernatants was determined by ELISA. (D,E) Freshly purified human monocytes were primed with $30 \mu \mathrm{g} / \mathrm{mL}$ LF-IC or PBS for $24 \mathrm{~h}$ followed by a further incubation with Jurkat (D) or Raji (E) in the presence of concentrations of GzB inhibitors (Z-AAD-CMK) for $18 \mathrm{~h}$. Cells were acquired and analyzed by FACS with PI staining. Results were expressed by the percentage of $\mathrm{CFSE}^{+} \mathrm{PI}^{+}$cell counts in $\mathrm{CFSE}^{+}$cell counts. (F) Freshly purified human monocytes were primed with $30 \mu \mathrm{g} / \mathrm{mL}$ LF-IC for $24 \mathrm{~h}$. Supernatant were removed and tumor cells were added followed by further incubation for $24 \mathrm{~h}$. In a parallel group, tumor cells were added without supernatant removement. GzB in supernatants was determined by ELISA. ${ }^{*} p<0.05,{ }^{* *} p<0.01,{ }^{* * *} p<0.001$. 


\subsection{Role of CD32a (Fc $\gamma$ RIIa) and Membrane-Bound CD14 in LF-IC Priming of Human Monocytes}

Membrane-bound CD14 (mCD14) is a co-receptor of TLR4, they function together as a receptor complex in monocytes for LF [19]. Consistent with our previous finding that LF-IC strongly activates human monocytes through co-ligation of Fc $\gamma$ RIIa (but not Fc $\gamma$ RI and Fc $\gamma$ RIII) and mCD14/TLR4 [19], GzB production by human monocytes under stimulation of LF-IC was significantly inhibited by $\alpha$ CD32a (Fc $\gamma$ RIIa), but not $\alpha \mathrm{CD} 64$ (Fc $\gamma$ RI) or $\alpha \mathrm{CD} 16$ (Fc $\gamma$ RIII), mAb (Figure $4 \mathrm{~A})$. Additionally, mAb against human CD14 almost completely abolished GzB production by LF-IC-primed monocytes (Figure 4A). Meanwhile, heparin, a carbohydrate capable of blocking LF-CD14 interaction, dose-dependently inhibited GzB production by human monocytes under LF-IC stimulation (Figure 4B).

A

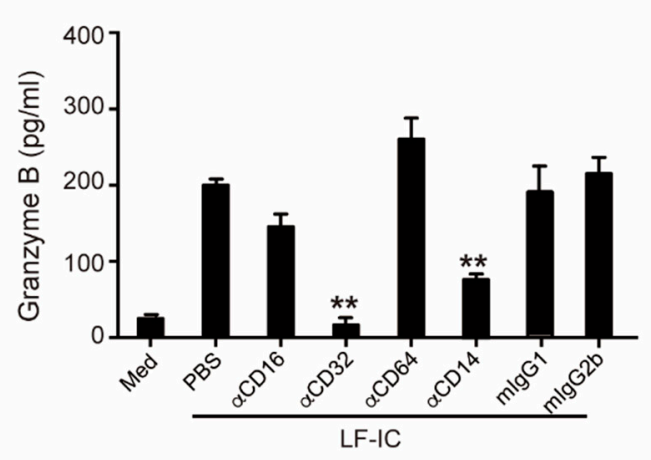

B

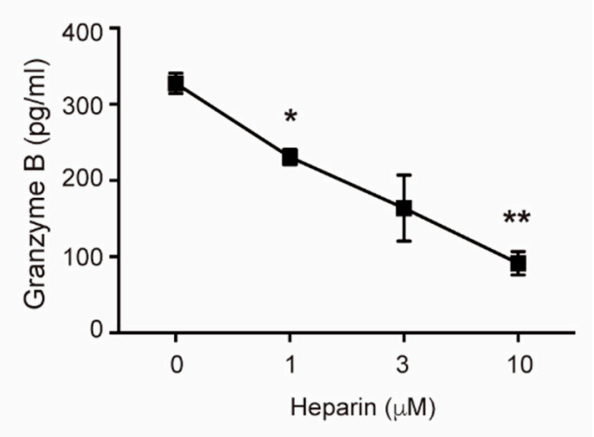

Figure 4. Crosslinking of Fc $\gamma$ RIIa and membrane-bound CD14 was essential for the tumoricidal activity empowerment to monocytes by LF-IC. (A) Freshly isolated human monocytes were primed with $30 \mu \mathrm{g} / \mathrm{mL}$ LF-IC for $48 \mathrm{~h}$ in the presence of $5 \mu \mathrm{g} / \mathrm{mL} \alpha \mathrm{CD} 16, \alpha \mathrm{CD} 32, \alpha \mathrm{CD} 64$ or $\alpha \mathrm{CD} 14$. mIgG1 and mIgG2b were included as isotype controls. (B) Monocytes were primed with $30 \mu \mathrm{g} / \mathrm{mL} \mathrm{LF}-\mathrm{IC}$ for $48 \mathrm{~h}$ in the presence of different concentration of heparin. GzB in supernatant was determined by ELISA. ${ }^{*} p<0.05,{ }^{* *} p<0.01,{ }^{* * *} p<0.001$.

\subsection{LF-IC Induces GzB Expression in Human Monocytes via the syk-PI3K-AKT Pathway}

Syk is a predominant kinase essential for signal transduction of immunoreceptor tyrosine-based activation motifs (ITAMs) in the cytoplasmic tail of Fc $\gamma$ Rs [33]. As shown in Figure 5A, LF-IC strongly induced phosphorylation of Akt, Syk and mTOR in human monocytes as evidenced by Western Blotting Assay (Figure 5A). Given also that PI3K was also induced in the same experiment (Figure 5A), it is highly likely that LF-IC elicited GzB production in human monocytes through the PI3K/Akt/mTOR pathway. This is supported by the results that specific inhibitors against Syk (R406), PI3K (LY294002), AKT (GSK690693) and mTOR (rapamycin) significantly decreased GzB expression in LF-IC-primed human monocytes (Figure 5B-E).

\subsection{Unresponsiveness of Murine Monocytes to LF-IC Stimulation}

Since mice do not have Fc $\gamma$ RIIa homologue, human Fc $\gamma$ RIIa transgenic (hFc $\gamma$ RIIa-TG) mice were employed for further investigation on whether LF-IC exhibits anti-tumor effect through augmentation of tumoricidal activity of monocytes/macrophages in vivo. Surprisingly, LF-IC-primed hFc $\gamma$ RIIa TG murine monocytes did not show tumoricidal activity against EL4 cells, a mouse leukemia cell line (Figure 6A). We also compared hFc $\gamma$ RIIa TG mice treated with, or without, LF-IC, for response to intravenous inoculated viable EL4 cells, no significant difference was observed in terms of time of survival (Figure 6B). 


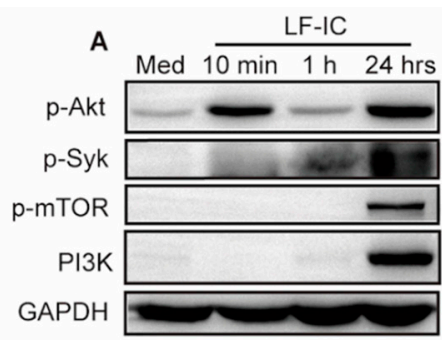

C

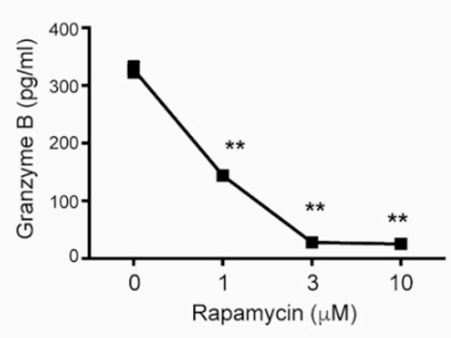

B

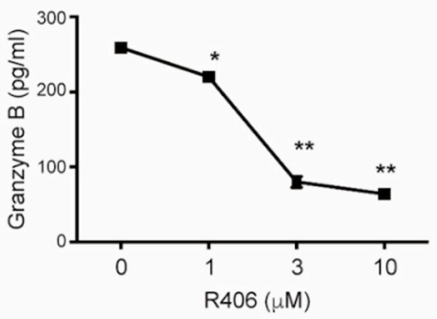

D

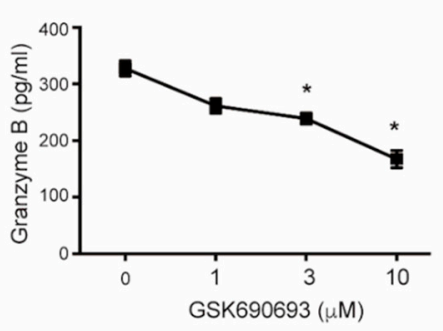

E

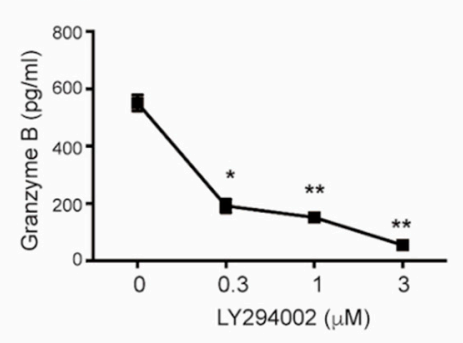

Figure 5. The signaling pathway for the tumoricidal activity empowerment to monocytes by LF-IC. (A) Freshly isolated human monocytes were stimulated with $30 \mathrm{mg} / \mathrm{mL}$ LF-IC for indicate time. The proteins were extracted and Western blotting analysis of the phosphorylation of syk, PI3K, Akt, and mTOR. (B-E) Monocytes were primed with $30 \mu \mathrm{g} / \mathrm{mL}$ LF-IC for $48 \mathrm{~h}$ in the presence of various concentration of kinase inhibitors, including SYK inhibitor (R406), mTOR inhibitor (rapamycin), AKT inhibitor (GSK690693) and PI3K inhibitor (LY294002). GzB in supernatant was determined by ELISA. ${ }^{*} p<0.05,{ }^{* *} p<0.01,{ }^{* * *} p<0.001$.

A

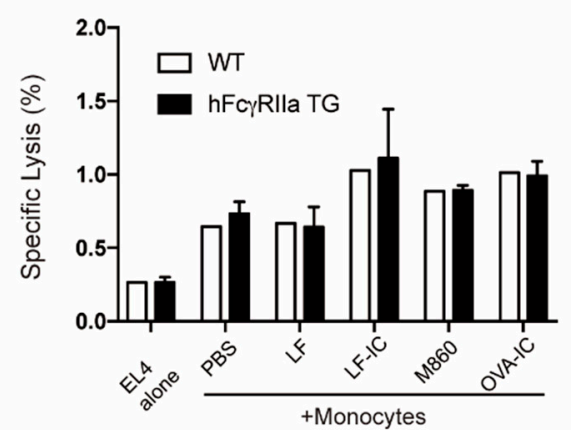

B

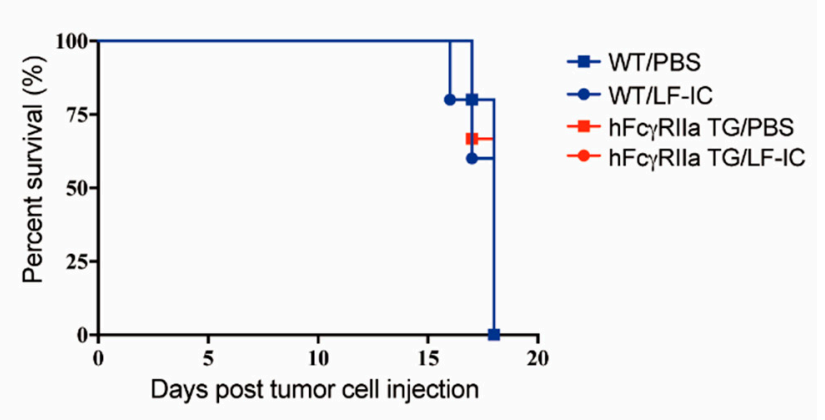

Figure 6. Unresponsiveness of mouse monocytes to LF-IC stimulation. (A) Freshly purified mouse monocytes of C57/BL6 wild type (WT) or hFc $\gamma$ RIIa transgenic mice (hFc $\gamma$ RIIa TG) were primed with $30 \mu \mathrm{g} / \mathrm{mL}$ LF, LF-IC, M860, OVA-IC for $24 \mathrm{~h}$, followed by further incubation with EL4 tumor cells for $18 \mathrm{~h}$. Cells were acquired and analyzed by FACS and results were expressed by the percentage of $\mathrm{CFSE}^{+} \mathrm{PI}^{+}$cell counts in $\mathrm{CFSE}^{+}$cell counts. ${ }^{*} p<0.05,{ }^{* *} p<0.01$. (B) WT or hFcgRIIa TG mice were inoculated with $10^{6}$ EL4 intravenously. Mice were treated with $100 \mu \mathrm{g}$ LF-IC (i.p.) at 1 day before inoculation and 1, 3 days post inoculation. Kaplan-Meier survival curves for all 6 mice/groups was shown.

\section{Discussion}

Tumorigenesis is a failure of immune surveillance towards malignant cells, therefore improving immune response is expected to have beneficial effect against cancers. LF exhibits functions of immunoregulation and anti-tumor protection [2,3]. However, its therapeutic efficacy is quite low, which has to be compensated by high dosage [8]. In this study, we demonstrate that combination of human LF and M860, a mouse monoclonal IgG1 antibody against human LF, renders human monocytes potent tumoricidal activity through Fc $\gamma$ RIIa-CD14/TLR4 co-ligation and induction of GzB. This strategy 
allows LF to achieve anti-tumor effect at significantly lower concentration. The Fc proportion of mouse IgG1 is able to bind human Fc $\gamma$ RIIa [34], which is a low affinity receptor for IgG in ICs. Humanized M860 with increased binding affinity for $h F c \gamma$ RIIa may further enhance the efficiency in facilitating the anti-tumor potential of low dose hLF in vivo.

Mouse monocytes transgenic for hFc $\gamma$ RIIa did not respond to LF-IC in the same way as human monocytes (Figure 6). Our recent data indicate that CD14/TLR4 complex on the surface of murine monocytic cells is also capable of binding hLF (not shown), thereby excluding the possibility that the unresponsiveness of hFc $\gamma$ RIIa-TG mouse monocytes to LF-IC was due to lack of binding with mouse CD14/TLR4. An important consideration here is that Fc $\gamma$ Rs expressed on the surface of mouse myeloid cells differ considerably from those of human monocytes. For instance, Fc $\gamma$ RIIb, an inhibitory $\mathrm{Fc} \gamma \mathrm{R}$, is expressed at a high level in mouse monocytes, but at a low level in human monocytes $[35,36]$. This could be a main reason for the poor response of mouse monocytes to LF-IC priming. Humanized mouse models would be needed for future investigation on anti-tumor effects of hLF in combination with M860 in vivo.

Among the cytokines released by LF-IC-primed human monocytes, TNF $\alpha$ could induce cell death of tumor cells. However, TNF $\alpha$ in LF-IC primed monocyte cultures was insufficient to induce cellular death of Jurkat and Raji cells. It has previously been documented that Jurkat cells were insensitive to TNF $\alpha$ or TRAIL [37]. Another possibility is that the amount of TNF $\alpha$ produced by LF-IC-primed monocytes was not large enough to achieve significant tumoricidal effects. Granzymes, a family of serine proteases expressed by cytotoxic cells, play important roles in protection against viral infection and cellular transformation. GzB is the most powerful member of the granzyme family. Our work identifies GzB as the major soluble mediator of the tumoricidal activity of LF-IC-primed monocytes. It has been shown that TLR8 agonist could boost antibody-dependent cell death (ADCC) through the induction of GzB [38]. Our data further emphasize that the interaction of TLRs with Fc $\gamma$ Rs could be beneficial for conditioning innate immune cells such as monocytes and macrophages for tumoricidal activity. Though cytotoxicity of GzB is not tumor-specific, receptors for LF, such as LRP, are well characterized tumor-associated antigens, which may help effective targeting of the LF-IC-primed monocytes $[39,40]$. In conclusion, our results on the tumoricidal activity of LF-IC-primed monocytes will expand the clinical cancer therapeutic application of LF and provide new research areas of tumor therapy.

\section{Materials and Methods}

\subsection{Reagents and Abs}

Human LF, LPS, heparin and OVA were taken from Sigma-Aldrich (STL, USA). Mouse IgG1 (ET901), IgG2b (MPC-11), mAbs against human Fc $\gamma$ RI (CD64, 10.1) or Fc $\gamma$ RIII (CD16, 3G8) were taken from BioLegend (San Diego, CA, USA). Anti-human Fc $\gamma$ RIIa mAb (CD32a, IV.3) was taken from STEMCELL Technologies (Vancouver, Canada). Mouse anti-human CD14 mAb (134620) and GzB ELISA kit were taken from R\&D (Minneapolis, MN, USA). GzB inhibitor (Z-AAD-CMK) was taken from Merck Millipore (Darmstadt, Germany). SYK inhibitor (R406), mTOR inhibitor (rapamycin), AKT inhibitor (GSK690693) and PI inhibitor (LY294002) were taken from Selleck (Houston, TX, USA). TNF $\alpha$ blocking antibody (Etanercept) was taken from AbMole (Houston, TX, USA).

\subsection{Peripheral Blood Monocyte Isolation and Cell Culture}

Peripheral blood monocytes (PBMs) were obtained from healthy individuals after informed consent in accordance with procedures approved by the human ethics committee of Soochow University. Monocytes were sorted from PBMCs using human $\alpha$ CD14 magnetic-labeled beads (MACS; Miltenyi Biotec) according to the manufacturer's instructions. The purity of monocytes obtained was around 95\%, as determined by flow cytometer with $\alpha$ CD14 mAb. Purified monocytes were cultured in RPMI-1640 medium supplemented with $10 \%(v / v)$ autologous serum, penicillin/streptomycin $(100 \mathrm{U} / \mathrm{mL})$, at $37^{\circ} \mathrm{C}$ 
and $5 \% \mathrm{CO}_{2}$. Human T-cell leukemia Jurkat cells. B-cell leukemia Raji cells and mouse leukemia EL4 cells were originally obtained from the American Type Culture Collection (ATCC). All cells were cultured in RPMI-1640 (Hyclone) supplemented with 10\% FBS (BI), penicillin/streptomycin (100 U/mL) at $37{ }^{\circ} \mathrm{C}$ with $5 \% \mathrm{CO}_{2}$.

\subsection{Mouse Monocyte Isolation}

Bone marrow cells from WT and hFc $\gamma$ RIIa TG mice were treated with red blood cell lysis buffer and resuspended in PBS at a density of $10^{8} / \mathrm{mL}$. Monocytes were enriched using EasySep ${ }^{\mathrm{TM}}$ Mouse Monocyte Isolation Kit (StemCell Technology, Vancouver, Canada). In brief, cell suspension incubated with selection cocktail including biotin labeled anti-CD3e, CD45R, Ly-6G, NK1.1, CD117 and Siglec F, followed by incubation with streptavidin coupled beads. The monocytes were purified using magnet depleting other cell population in bone marrow.

\subsection{Anti-Tumor Effects}

Freshly purified human monocytes or mouse monocytes were seeded at a density of $10^{5}$ cells per well in a 96 well plate and primed with stimulators for 24 h. $2 \times 10^{4}$ CFSE labeled Jurkat or Raji were later added and co-cultured with primed human monocytes for 18 h. $2 \times 10^{4}$ CFSE labeled EL4 cells were added and co-cultured with freshly purified mouse monocytes for $18 \mathrm{~h}$. Cells were acquired and analyzed by FACS with PI (Propidium Iodide) staining. Results were expressed by the percentage of $\mathrm{CFSE}^{+} \mathrm{PI}^{+}$cell counts in $\mathrm{CFSE}^{+}$cell counts.

\subsection{ELISA}

The concentration of human Granzyme B in the culture supernatant was determined using ELISA kits (Minneapolis, R\&D, USA). Cell supernatants were collected and centrifuged at 10,000 g to clear cellular debris. ELISAs were performed according to the respective manufacturer protocols.

\subsection{Real-Time RT-PCR}

RNA was isolated from cells with the Total RNA Kit II (OMEGA, Norcross, GA, USA) and subsequently reversely transcribed into cDNA using an oligo (dT) primer (Takara, JP). For mRNA-level analysis, cells were lysed at the indicated time points, after which mRNA extraction was performed. Q-PCR (Life Technology, USA) was performed using SYRB green (Takara, JP) and primer pairs as listed in Table 1.

Table 1. Primers for indicated genes.

\begin{tabular}{ccc}
\hline & Forward $\left(\mathbf{5}^{\prime} \mathbf{-} \mathbf{3}^{\prime}\right)$ & Reverse $\mathbf{( 5}^{\prime} \mathbf{s}^{\prime} \mathbf{)}$ \\
\hline hGAPDH & GAGTCAACGGATTTGGTCGT & TTGATTTTGGAGGGATCTCG \\
\hline $\mathrm{hGzA}$ & AACCAGGAACCATGTGCCAA & GGCTTCCAGAATCTCCATTGC \\
\hline $\mathrm{hGzB}$ & GAGCAAGGAGGAAACAACAGC & GGCCCCCAAGGTGACATTTA \\
\hline $\mathrm{hGzH}$ & ATGCTACTGCAGGGGGACT & TCAGGCCCAGAGGAAGGTTA \\
\hline $\mathrm{hGzK}$ & CCCTGCGAGAAGTCACTGTT & CCCCCTGAGTCACCCTTACA \\
\hline $\mathrm{hGzM}$ & GTCAGTAGCTCCTTTGGGACC & GGCTGTTGTTACACATGCGG \\
\hline
\end{tabular}

\subsection{Tumor Models}

WT or hFc $\gamma$ RIIa TG mice were obtained from Jackson Laboratory (Bar Harbor, ME, USA) and bred with C57/BL6 mice for more than 10 generations. WT and hFc $\gamma$ RIIa TG $(n=12)$ were inoculated with $10^{6}$ EL4 intravenously. Mice were treated with $100 \mu \mathrm{g}$ LF-IC or PBS i.p. at the day before inoculation and 1, 3 days post inoculation. Survival of the animals were monitored for 20 days post inoculation. 


\subsection{Western Blotting Analysis}

Cells were lysed in lysis buffer. After centrifugation at $12,000 \mathrm{~g}$ for $15 \mathrm{~min}$ at $4{ }^{\circ} \mathrm{C}$, the supernatant was collected, and its protein concentration was determined using BCA Protein Assay Kit (Pierce, Rockford, IL, USA). $30 \mu \mathrm{g}$ proteins for each sample, were separated on $10 \%$ SDS-PAGE and transferred onto polyvinylidene difluorid (PVDF) membranes (Millipore, Bedford, MA, USA), followed by being blocked with 5\% bovine serum albumin in PBST (0.1\% Tween-20 in PBS) for $1 \mathrm{~h}$. The membranes were incubated with anti-p-Syk, p-PI3K, p-Akt, p-mTOR and $\beta$-actin (Cell signaling technology, Danvers, MA, USA) at $4{ }^{\circ} \mathrm{C}$ overnight followed by incubation with horseradish peroxidase-conjugated secondary antibody for $1 \mathrm{~h}$ at room temperature. The blots were developed by enhance chemiluminscence detection regents (Pierce, Rockford, IL, USA).

\subsection{Study Approval}

This study was approved by the Ethics Committees of Soochow University, Suzhou, China. The methods were carried out in accordance with the guidelines of Soochow University. Written informed consent was obtained from all participants prior to inclusion in the study.

\subsection{Statistical Analysis}

Data were expressed as the mean \pm SEM of at least three independent experiments. The student's $t$-test was used to compare differences among groups by using GraphPad Prism 5.0 software and values at $p<0.05$ were considered significant.

\section{Conclusions}

Data presented here demonstrate that IC between hLF and M860, an anti-hLF mAb, renders human monocytes tumoricidal activiy via induction of secreted GzB. LF-IC is able to colligate CD14/TLR4 and Fc $\gamma$ RIIa on the surface of human monocytes, thereby triggering the Syk-PI3K-AKT-mTOR pathway leading to GzB production. Our results may help to expand cancer therapeutic application of low dosage LF.

Author Contributions: Supervision, X.-M.G., F.-Y.G.; Investigation, Y.L., J.L., X.-H.P., Z.G., Z.-H.M., S.-Y.M., H.-M.W., F.-Y.G.; Data Curation, H.-L.D., F.-Y.G.; Writing—Original Draft Preparation, F.-Y.G.; Writing—Review \& Editing, X.-M.G.

Funding: This study was supported by grants from Ministry of Science and Technology (2017YFA0104502), grants from National Foundation of Nature Science of China (31570868), Foundation for Application and Fundamental Research of Healthcare, Suzhou (SYS201667).

Conflicts of Interest: The authors declare no conflict of interest.

\section{References}

1. Vogel, H.J. Lactoferrin, a bird's eye view. Biochem. Cell Biol. 2012, 90, 233. [CrossRef] [PubMed]

2. Damiens, E.; El Yazidi, I.; Mazurier, J.; Duthille, I.; Spik, G.; Boilly-Marer, Y. Lactoferrin inhibits G1 cyclin-dependent kinases during growth arrest of human breast carcinoma cells. J. Cell. Biochem. 2015, 74, 486-498. [CrossRef]

3. Mader, J.S.; Salsman, J.; Conrad, D.M.; Hoskin, D.W. Bovine lactoferricin selectively induces apoptosis in human leukemia and carcinoma cell lines. Mol. Cancer 2005, 4, 612. [CrossRef] [PubMed]

4. Hoedt, E.; Hardivillé, S.; Mariller, C.; Elass, E.; Perraudin, J.P.; Pierce, A. Discrimination and evaluation of lactoferrin and delta-lactoferrin gene expression levels in cancer cells and under inflammatory stimuli using TaqMan real-time PCR. Biometals 2010, 23, 441-452. [CrossRef] [PubMed]

5. Benaïssa, M.; Peyrat, J.P.; Hornez, L.; Mariller, C.; Mazurier, J.; Pierce, A. Expression and prognostic value of lactoferrin mRNA isoforms in human breast cancer. Int. J. Cancer 2005, 114, 299-306. [CrossRef] [PubMed]

6. Lee, S.H.; Sang, W.P.; Pyo, C.W.; Yoo, N.K.; Kim, J.; Choi, S.Y. Requirement of the JNK-associated Bcl-2 pathway for human lactoferrin-induced apoptosis in the Jurkat leukemia T cell line. Biochimie 2009, 91, 0-108. [CrossRef] [PubMed] 
7. Xu, X.X.; Jiang, H.R.; Li, H.B.; Zhang, T.N.; Zhou, Q.; Liu, N. Apoptosis of stomach cancer cell SGC-7901 and regulation of Akt signaling way induced by bovine lactoferrin. J. Dairy Sci. 2010, 93, 2344-2350. [CrossRef] [PubMed]

8. Jiang, R.; Lönnerdal, B. Bovine lactoferrin and lactoferricin exert antitumor activities on human colorectal cancer cells (HT-29) by activating various signaling pathways. Biochem. Cell Biol. 2016, 95, 1. [CrossRef] [PubMed]

9. Birgens, H.S.; Karle, H.; Hansen, N.E.; Ostergaard, K.L. Lactoferrin receptors in normal and leukaemic human blood cells. Scand. J. Haematol. 2010, 33, 275-280. [CrossRef] [PubMed]

10. Damiens, E.; Mazurier, J.; Yazidi, I.E.; Masson, M.; Duthille, I.; Spik, G.; Boilly-Marer, Y. Effects of human lactoferrin on NK cell cytotoxicity against haematopoietic and epithelial tumour cells. Biochim. Biophys. Acta 1998, 1402, 277-287. [CrossRef]

11. Ben, A.; Qian, G.; Mark, W. Bovine lactoferrin structures promoting corneal epithelial wound healing in vitro. Investig. Ophthalmol. Vis. Sci. 2011, 52, 2719-2726.

12. Mario, F.D.; Aragona, G.; Bò, N.D.; Cavestro, G.M.; Cavallaro, L.; Iori, V.; Comparato, G.; Leandro, G.; Pilotto, A.; Franzè, A. Use of bovine lactoferrin for Helicobacter pylori eradication. Dig. Liver Dis. 2003, 35, 706-710.

13. Valenti, P.; Antonini, G. Lactoferrin: An important host defence against microbial and viral attack. Cell. Mol. Life Sci. Cmls 2005, 62, 2576-2587. [CrossRef] [PubMed]

14. Sánchez, L.; Calvo, M.; Brock, J.H. Biological role of lactoferrin. Haematologia 1985, 18, 3. [CrossRef] [PubMed]

15. Bo, L.N.; Rulan, J.; Xiaou, D. Bovine lactoferrin can be taken up by the human intestinal lactoferrin receptor and exert bioactivities. J. Pediatric Gastroenterol. Nutr. 2011, 53, 606-614.

16. Debanne, M.T.; Regoeczi, E.; Sweeney, G.D.; Krestynski, F. Interaction of human lactoferrin with the rat liver. Am. J. Physiol. 1985, 248, G463. [CrossRef]

17. Bennett, R.M.; Davis, J. Lactoferrin binding to human peripheral blood cells: An interaction with a B-enriched population of lymphocytes and a subpopulation of adherent mononuclear cells. J. Immunol. 1981, 127, 1211.

18. Hu, X.M.; Xu, Y.R.; Yan, R.; Sun, S.L.; Dong, H.L.; Wang, J.; Gao, X.M. A Novel Murine Anti-Lactoferrin Monoclonal Antibody Activates Human Polymorphonuclear Leukocytes through Membrane-Bound Lactoferrin and TLR4. Biomed Res. Int. 2015, 10, 1-11. [CrossRef]

19. Hu, L.; Hu, X.; Long, K.; Gao, C.; Dong, H.L.; Zhong, Q.; Gao, X.M.; Gong, F.Y. Extraordinarily potent proinflammatory properties of lactoferrin-containing immunocomplexes against human monocytes and macrophages. Sci Rep 2017, 1, 1-4230. [CrossRef]

20. Alberto, M.; Silvano, S.; Massimo, L.; Paola, A.; Antonio, S. Macrophage polarization: Tumor-associated macrophages as a paradigm for polarized M2 mononuclear phagocytes. Trends Immunol. 2002, 23, 549-555.

21. Gao, C.H.; Dong, H.L.; Tai, L.; Gao, X.M. Lactoferrin-Containing Immunocomplexes Drive the Conversion of Human Macrophages from M2- into M1-like Phenotype. Front. Immunol. 2018, 9, 37. [CrossRef] [PubMed]

22. Mantovani, A.; Sica, A. Macrophages, innate immunity and cancer: Balance, tolerance, and diversity. Curr. Opin. Immunol. 2010, 22, 231-237. [CrossRef] [PubMed]

23. O'Sullivan, T.; Saddawi-Konefka, R.; Vermi, W.; Koebel, C.M.; Arthur, C.; White, J.M.; Uppaluri, R.; Andrews, D.M.; Ngiow, S.F.; Teng, M.W.; et al. Cancer immunoediting by the innate immune system in the absence of adaptive immunity. J. Exp. Med. 2012, 209, 1869-1882. [CrossRef] [PubMed]

24. Antonio, S.; Vincenzo, B. Altered macrophage differentiation and immune dysfunction in tumor development. J. Clin. Investig. 2007, 117, 1155-1166.

25. Qian, B.Z.; Pollard, J.W. Macrophage Diversity Enhances Tumor Progression and Metastasis. Cell 2010, 141, 39-51. [CrossRef] [PubMed]

26. Griffith, T.S.; Wiley, S.R.; Kubin, M.Z.; Sedger, L.M.; Maliszewski, C.R.; Fanger, N.A. Monocyte-mediated tumoricidal activity via the tumor necrosis factor-related cytokine, TRAIL. J. Exp. Med. 2015, 189, 1343-1354. [CrossRef]

27. Lindholm, P.F.; Sivapurapu, N.; Jovanovic, B.; Kajdacsy, B.A. Monocyte-Induced Prostate Cancer Cell Invasion is Mediated by Chemokine ligand 2 and Nuclear Factor-кB Activity. J. Clin. Cell. Immunol. 2015, 6, 308. [CrossRef] [PubMed]

28. Lauren, B.E.; David, S.; Fang, T.; Hyung-Gu, K.; Takushi, N.; César, M.O.F.; Anna, M.; Aaronson, S.A.; Lee, S.W. Expression of the p53 target CDIP correlates with sensitivity to TNF $\alpha$-induced apoptosis in cancer cells. Cancer Res. 2012, 72, 2373. 
29. Wang, C.Y.; Mayo, M.W.; Korneluk, R.G.; Goeddel, D.V.; Baldwin, A.S., Jr. NF-kappaB antiapoptosis: Induction of TRAF1 and TRAF2 and c-IAP1 and c-IAP2 to suppress caspase-8 activation. Science 1998, 281, 1680-1683. [CrossRef]

30. Zhong, Q.; Gong, F.Y.; Gong, Z.; Hua, S.H.; Gao, X.M. IgG Immunocomplexes Sensitize Human Monocytes for Inflammatory Hyperactivity via Transcriptomic and Epigenetic Reprogramming in Rheumatoid Arthritis. J. Immunol. 2018, 200, 3913-3925. [CrossRef]

31. Russell, J.H.; Ley, T.J. Lymphocyte-mediated cytotoxicity. Annu. Rev. Immunol. 2002, 20, 323-370. [CrossRef]

32. Mahrus, S.; Craik, C.S. Selective Chemical Functional Probes of Granzymes A and B Reveal Granzyme B Is a Major Effector of Natural Killer Cell-Mediated Lysis of Target Cells. Chem. Biol. 2005, 12, 567-577. [CrossRef] [PubMed]

33. Parren, P.W.; Warmerdam, P.A.; Boeije, L.C.; Arts, J.; Westerdaal, N.A.; Vlug, A.; Capel, P.J.; Aarden, L.A.; Van de Winkel, J.G. On the interaction of IgG subclasses with the low affinity Fc gamma RIIa (CD32) on human monocytes, neutrophils, and platelets. J. Clin. Investig. 1992, 90, 1537-1546. [CrossRef] [PubMed]

34. Gillis, C.M.; Jönsson, F.; Mancardi, D.A.; Tu, N.; Beutier, H.; Van Rooijen, N.; Macdonald, L.E.; Murphy, A.J.; Bruhns, P. Mechanisms of anaphylaxis in human low-affinity IgG receptor locus knock-in mice. J. Allergy Clin. Immunol 2017, 139, 1253-1265. [CrossRef] [PubMed]

35. Veri, M.-C.; Gorlatov, S.; Li, H.; Burke, S.; Johnson, S.; Stavenhagen, J.; Stein, K.E.; Bonvini, E.; Koenig, S. Monoclonal antibodies capable of discriminating the human inhibitory Fc $\gamma$-receptor IIB (CD32B) from the activating Fc $\gamma$-receptor IIA (CD32A): Biochemical, biological and functional characterization. Immunology 2007, 121, 392-404. [CrossRef]

36. Yuasa, T.; Kubo, S.; Yoshino, T.; Ujike, A.; Matsumura, K.; Ono, M.; Ravetch, J.V.; Takai, T. Deletion of fcgamma receptor IIB renders H-2(b) mice susceptible to collagen-induced arthritis. J. Exp. Med. 1999, 189, 187-194. [CrossRef] [PubMed]

37. Fas, S.C.; Sven, B.; Yun, Z.J.; Marco, G.; Treiber, M.K.; Ulrich, M.; Krammer, P.H.; Min, L.W. Wogonin sensitizes resistant malignant cells to TNFalpha- and TRAIL-induced apoptosis. Blood 2006, 108, 3700-3706. [CrossRef]

38. Saranya, E.; Kavin, F.; Vikram, S.; Huiqing, F.; Li, R.; Shalini, G.; Brenda, R.; Xiaokui, M.; Carolyn, C.; Edward, B. Granzyme B expression is enhanced in human monocytes by TLR8 agonists and contributes to antibody-dependent cellular cytotoxicity. J. Immunol. 2015, 194, 2786-2795.

39. Ji, Z.S.; Mahley, R.W. Lactoferrin binding to heparan sulfate proteoglycans and the LDL receptor-related protein. Further evidence supporting the importance of direct binding of remnant lipoproteins to HSPG. Arter. Thromb. A J. Vasc. Biol. 1994, 14, 2025. [CrossRef]

40. Duchardt, F.; Ruttekolk, I.W. A cell-penetrating peptide derived from human lactoferrin with conformation-dependent uptake efficiency. J. Biol. Chem. 2010, 284, 36099-36108. [CrossRef]

Sample Availability: Samples of the compounds are available from the authors.

(C) 2019 by the authors. Licensee MDPI, Basel, Switzerland. This article is an open access article distributed under the terms and conditions of the Creative Commons Attribution (CC BY) license (http://creativecommons.org/licenses/by/4.0/). 